$196 / 2$ PPPL-1960 (1)

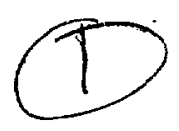

PPPL-1960

\title{
$\pm .7312$
}

THE DIFFUSIVITY OF HYDROGEN ISOTOPES IN LIQUID

LITHIUM AND IN SOLID YTTRIUM

\section{$\mathbf{N}$ \\ By \\ R.E. Buxbaum and E.F. Johnson}

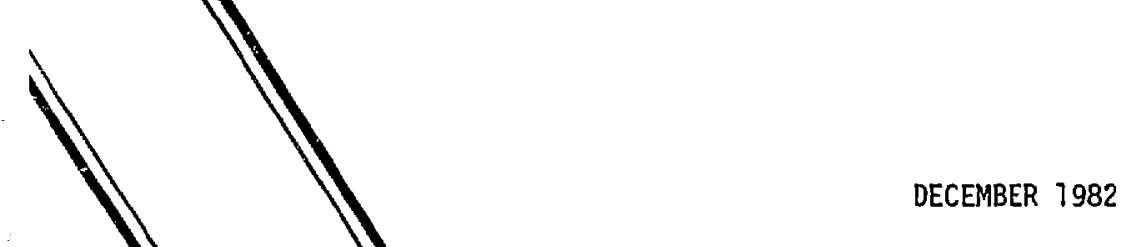

\section{N \\ M \\ L

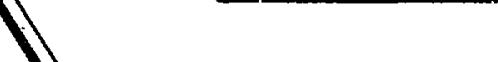 \\ PLASMA \\ PHYSICS \\ LABORATORY}

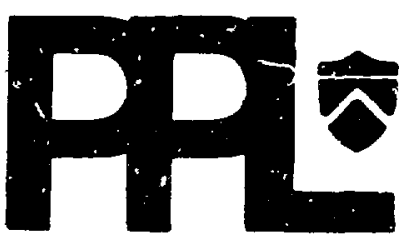

PRINCETON UNIVERSITY

PRINCETON, NEW JERSEY

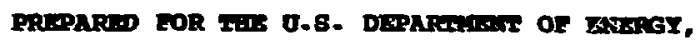

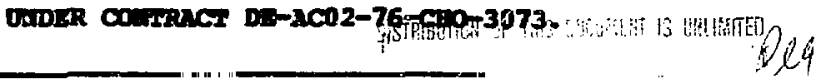


THE DIFFUSIVITY OF HYDROGEN ISOTOPES IN LIQUID

LITHIUM AND IN SOLID YTTRIIM

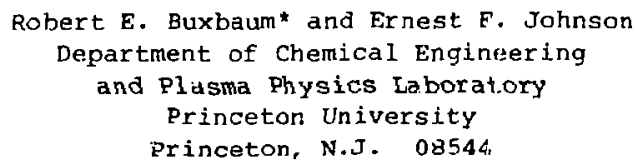

\section{ABSTRACT}

The separation of tritium from liquid lithium at low conc trations is an important problem for fusion technology. One of the most promis ng terhniques for effecting this separation involves sorbing the tritium on solid yttrium metal. Reliable diffusivity data are essential for a realistic evaluation of this technique.

This paper reviews the bodies of relevant experimental diffus ity data, i.e., data on protium diffusion in liquid lithium, protium diffusior in solid yttrium, and the isotope effect in metals. On the basis of these data and in the light of standard correlations, the following equations are proposed for the diffusivity of tritium in liquid lithium and in solja yterium respectively:

$$
\log _{10} \mathrm{D}_{\mathrm{T}-\mathrm{Li}}=-9.038+1.737 \log _{10} \mathrm{~T}-110 / \mathrm{T} \mathrm{cm}^{2} / \mathrm{s}
$$

and

$$
\log _{10} \mathrm{D}_{\mathrm{T}-\mathrm{Y}}=-2.391-2558 / \mathrm{T}
$$

These equations show that the geometries of yttrium systems for tritium gettering in fusion reactors are of quite practicable dimensions. 
I. SCOPE

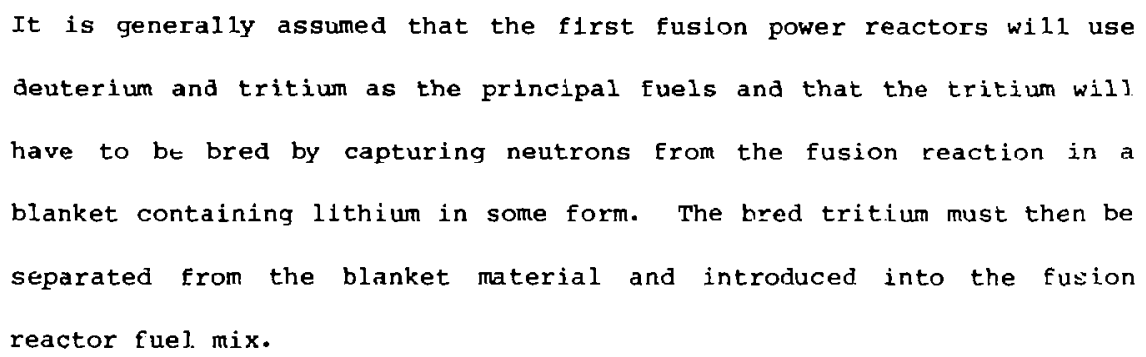

As demonstrated by Buxbaum and Johnson (1980), from many standpoints, the simplest and most attractive breeding medium is liquid lithium, and the most attractive tritium recovery medium is solid yttrium metal. However, in the absence of experimental aata on test loops, the practicability of this separation system can be gauged only from paper studies, and such studies will be critically dependent on reliable information on the relevant diffusivity properties. This paper presents the best available information on these properties.

II. THE DIFFUSIVITY OF TRITILM IN LIQUID LITHIUM Cussler (1976) has generalized that the diffusivity of any solute in any liquid solvent is a constant approximating $0.7 \times 10^{-5} \mathrm{~cm}^{2} / \mathrm{s}$. While this assertion tends to be accurate within an order of magnitude, a more reliable estimate, polymeric liquids excepted, may be made on the basis of the Nernst-Einstein (Langevin, 1908) equation. A convenient simplification of this equation is the stokes-Einstein equation (Einstein, 1905) viz.,

$$
D=k T / 6 \pi \tilde{x}_{\mu}
$$


where $k$ is Boltzman's constant, $1.3805 \times 10^{-13} \mathrm{~N}-\mathrm{cm} / \mathrm{K}$; $\mathrm{T}$ is absolute temperature, $K_{i} \|$ is the solvent viscosity, $\mathrm{N}-\mathrm{s} / \mathrm{cm}^{2} ;$ and $\bar{r}$ is the effective radius of the diffusing species $\mathrm{cm}$. For relatively spherical molecules,

$$
\bar{r} \simeq\left(3 V / 4 \pi N_{A v}\right)^{1 / 3}
$$

where $V$ is the molecular volume at the normal boiling point, $\mathrm{cm}^{3} / \mathrm{g}$ mol; and $\mathrm{N}_{\mathrm{AV}}$ is Avogadro's numbei, $6.02 \times 10^{23}$ molecules/g mol. For nonspherical molecules, the effective radii may be determined from their pseudo-steady hydrcdynamic mobilities (Brenner, 1974).

The effective radius of the lithium hydride molecule, when calculated by Eq. (2), is found to be 1.7 \&. This radius, when inserted into Eq. (1). does not correlate well with the experiment, presumably because the diffusing species is not the lithium hydride molecule. Rather lydrogen diffuses in liquid lithium as $\mathrm{Li}_{2} \mathrm{H}$ molecules, $\mathrm{Li}_{3} \mathrm{H}$ molecules, or possibly as larger conglomerates.

For smaller molecules diffusing in non-polymeric liquids, the most often used correlations are those of wilke and chang (1955) and scheibel (1954). Their correlations may be expressed as

$$
\mathbf{D}=7.4 \times 10^{-8} \frac{\left(M_{B}\right)^{1 / 2} T}{L_{a}^{*} 0.6}
$$

and 


$$
D=8.2 \times 10^{-8} \frac{T}{\mu^{*}} \frac{\frac{3 V_{B}}{V_{A}}}{\left(V_{A}\right)^{1 / 3}},
$$

respectively. Here $v_{A}$ and $v_{B}$ are the molecular volumes of the solute and the solvent respectively, $\mathrm{cm}^{3} /$ mole; $^{M_{B}}$ is the solvent's molecular weight, $g / m o l ; T$ is absulute temperature, $K$; and $\mu^{*}$ is the solvent viscosity in centipoise. The experimental data in both of these references are seen to support the assertion from theoretical calculations (e.g., Batchelor, 1976) that liquid diffusivity is proportional to absolute temperature divided by viscosity, poise, and

$$
\mathbf{D}=\frac{\mathbf{a T}}{\mathrm{L}}
$$

where $a$ is a constant determined by temperature-independent properties of tre solute-solvent pair.

Equation 5, which has been found to be universally applicable to nonpolymer systems, can be used to extrapolate the available, hightemperature protium-lithium diffusivity data into the low-temperature regime. Furthermore, since nolecular volume is largely independent of isotope mass, Eqs. (1), (3), and (4) each predict that the constant, a, in Eq. (5) is isotope independent. Thus, at any given temperature, the Aiffusivity of all isotopes of hydrogen in liquid lithium should be the same. 
We are now in a position to fit experimental data to Eg. (5). The viscosity of liquid is reliably reported by shpil'rain et al. (1965), over the range 185 to $1000^{\circ} \mathrm{C}$, as

$$
\log _{10} \mu^{*}=1.4936-0.7368 \log _{10} T+109.95 / \mathrm{T} \text {. }
$$

It is not expected that the lithium viscosity is affected by small hydrogen concentrations. Equations (5) and (6), when fitted with Alire's (1976) high-temperature, lithsum-protium diffusion data (Table 1), produce a broad range diffusivity correlation applicable to all hydrogen isotopes. The value of $a$ is found to be $2.86 \times$ $10^{-10} \mathrm{erg} / \mathrm{cmk}$ with a standard deviation of $18 \%$. The diffusivity of hyarogen isotopes in liquid lithium thus calculated is

$$
\log _{10}(D \pm 188)=-9.038+1.737 \log _{10} T-110 / \mathrm{T}
$$

While Eq. (7), strictly speaking, is only applicable to the diffusion of hydrogen isotopes in pure lithium, it may be used as an aid in predicting the diffusivity of hydrogen isotopes in molten lithium-rich alloys of interest to fusion technology: lithium-lead and Lithiumaluminum. An equivalent radius or volume for the diffusing lithium hydride species may be calculated by combining Eas. (7) and (6; with Eq. (1), (3), or (4). For example, $\bar{I}$ as calculated from Eqs. (7), (6), and (1), is 2.65 \&. Given this value, assumed constant for lithium-rich systems, and a vıscosity relation for the molten alloy in question, the calculation of the diffusivity of hydrogen isotopes in that alloy is straight forward. 
Table 1: Diffusion Coefficients of $H_{2}$ in Lithium at High Temperatures, from Alire (1976).

\begin{tabular}{lcc}
$\begin{array}{c}\text { Temperature } \\
\left({ }^{\circ} \mathrm{C}\right)\end{array}$ & $\begin{array}{c}\text { Pressure } \\
(\mathrm{Pa})\end{array}$ & $\begin{array}{c}\text { Diffusicun } \\
\text { Coefficient } \\
\left(\mathrm{cm}^{2} / \mathrm{s}\right)\end{array}$ \\
\hline 800 & 575.0 & $0.97 \times 10^{-4}$ \\
800 & 15.6 & $1.10 \times 10^{-4}$ \\
800 & 15.6 & $0.95 \times 10^{-4}$ \\
800 & 1097. & $1.11 \times 10^{-4}$ \\
800 & 1097. & $1.04 \times 10^{-4}$ \\
800 & 1097. & $1.33 \times 10^{-4}$ \\
800 & 1097. & $1.25 \times 10^{-4}$ \\
805 & 1097. & $1.30 \times 10^{-4}$ \\
\hline 805 & 575. & $1.33 \times 10^{-4}$ \\
875. & 575. & $1.54 \times 1-^{-4}$ \\
\hline
\end{tabular}


III. THE DIFFUSIUTTY OF HYDROGEN ISOTOPES IN YTTRIUM The full body of experimental data for low concentration hydrogen diffusion in yttrium metal is presented in Fig. 1. While we expect that hydrogen diffuses through hexagonal-close-packed yttrium metal by an interstitial mechanism, this assumption leads to the prediction of an Arrhenius' law temperature-dependence for diffusion. The data in Fig. I do not immediately support this expectation.

The most striking feature of Fig. 1 is the fact that those values of the aiffusivity coefficient which were measured by the rate of observed vacuum desorption, Voroboyov et al. (1976) and Talbot et al. (1979), are much lower than those values of the diffusion coefficient measured by neutron scattering or quantitative analysis. The simplest explanation for this discrepancy is that surface resistance, the hydrogen recombination reaction, for example, reduced the apparent dif usivity in the vacuun desorption experiments. This hypothesis, suggested by Buxbaum et al. (1990) and Talbot (1981), is supported by the recombination rate experiments of Jung $(1973-4)$ and also by the fact that the apparent diffusivity measured by Talbot et al. (1980) increased when hydrogen was added to the sweep gas.

close agreement is found among experiments where no surface resistance was possible: Carlson et al. (1966). Fresius et al. (1972), and Fresius et al. (1976). Using a least squares fit for the data in these references, the diffusivity of protium in yttrium is calculated to be $\log _{10}(\mathrm{D} \pm 208)=-2.425-2402 / \mathrm{T}$.

This equation is represented by the solid line in Fig. 1. 
IV. ISOTOPE EFFECTS IN YTTRIUM-HYDROGEN

At least two different approaches can be used to predict the isotope effect in yttrium-hydrogen diffusion. One approach is purely classical; as described by zener (1950),

$$
\begin{aligned}
\frac{D_{H-Y}}{D_{T-Y}} & =\frac{\sqrt{M_{1}}}{\sqrt{M_{3}}} \\
& =\frac{1}{\sqrt{3}}
\end{aligned}
$$

where $M_{1}$ and $M_{3}$ are the atomic masses of protium and tritium respectively.

A compendiun djffusion study by Volkyl and Alefield (1978) shows many striking departures from the square-root-of-mass dependence of $\mathrm{Eq}$. (9). The most pronounced of these effects is seen at temperatures below $400^{\circ} \mathrm{C}$, and for hydrogen isotopes in the following host metals: palladium, niobium, asci vanadium. Eqs. ( 8 ) and (9) predict that the diffusivity of tritium in yttriun is

$$
\log _{10} D( \pm 308)=-2.663-2402 / T \text {. }
$$

Anothex approach, described by Buxbaum (1981), predicts a diffusivity relation which should be applicable over a wider range of temperatures. The author assumes the entire isotope offat is due to differences in the zero-point-energy cretcisution to the activation energy for diffusion. 


$$
\frac{\mathbf{D}_{\mathrm{H}-\mathrm{Y}}}{\mathbf{D}_{\mathrm{T}-\mathrm{Y}}}=\exp \frac{-\beta\left(\Delta \mathrm{H}_{1}-\Delta \mathrm{H}_{3}\right)}{\mathrm{R} \mathrm{T}_{\mathrm{m}}}-\exp \frac{\left(\Delta \mathrm{H}_{1}-\Delta \mathrm{H}_{3}\right)}{\mathrm{RT}}
$$

where $T_{m}$ is the melting temperature of yttrium, $1768 \mathrm{~K} ; \Delta \mathrm{H}_{1}$ is the activation energy for protilim diffusion in yttrium, $7.65 \times 10^{-13}$ erqs/atom (10990 cal/g-atom); $\beta$ is a dimensionless elastic constant, approximately equal to $.35 ;$ and $\left(\mathrm{H}_{1}-\mathrm{H}_{3}\right)$ is the zero-point energy difterence for protium and tritium in yttrium. For a sinusoidal, onedimensional potential,

$$
\begin{aligned}
\left(\Delta_{1}-\Delta H_{3}\right) & =\frac{h}{2 \alpha} \sqrt{\left(H_{1}+\Delta_{3}^{H}\right)}\left(1 / \vec{M}_{1}-1 / \vec{M}_{3}\right. \\
& =4.95 \times 10^{-14} \text { ergs/atom } \\
& =710 \mathrm{cal} / 9 \text {-atom. }
\end{aligned}
$$

Here $h$ is Planck's constant, $6.657 \times 10^{-27} \mathrm{erg} s ; M_{1}$ and $M_{3}$ are the atomic masses of protium and tritium, $1.66 \times 10^{-24}$ and $4.98 \times$ $10^{-24} \mathrm{~g} /$ atom respectively; and $\alpha$ is the distance between nearestneighbor interstitial sites in yttrium, $297 \times 10^{-8} \mathrm{~cm}$.

From Eqs. (8), $(19)$, ard (12), the diffusivity of tritium in yttrium is predicted to be

$$
\begin{aligned}
& D=(0.00406 \pm 0.0006) \exp [(11700 \pm 800) / \mathrm{RT}] \\
& \log _{10} D( \pm 208)=-2.391-2558 / \mathrm{T} .
\end{aligned}
$$


One important application of $\mathrm{Eg}$. (13) is in choosing the thickness Eor an absorber column using yttriium metal as a tritium getter. As an example calculation, consider an absorber which operates for 24 hours at $200^{\circ} \mathrm{C}$ before being regenerated. At this temperature

$$
D_{Y-T}=1.60 \times 10^{-8}
$$

The one-dimensional penetration depth $\eta$, $\mathrm{cm}$, is given by

$$
n=\sqrt{\pi \mathrm{Dt}}
$$

where $t$ is the sorption time, s. For the conditions specifiec above,

$$
\begin{aligned}
\eta & =\sqrt{ }(3.14)\left(1.60 \times 10^{-8}\right)(86400) \\
& =0.065 \mathrm{~cm} .
\end{aligned}
$$

One may now size the metal thickness by setting it equal to some factor of the penetration depth, preferably one to two times $\eta$ (Buxbaum, 1981). Sheet thicknesses, calculated in this manner, are well within the range available from commercial distributors.

\section{ATKNOWLEDGMNT}

This work was supported in part by the U.S. Department of Energy Contract No. DE-AC02-76-CHO-3073. 
Alire, R.M.. J. Chem. Phys. 1976, 65, 1134-37.

Batchelor, G.K., "Theoretical and Applied Mechanics," eỏ. W. Keiter, North Holland, Amsteräam, 1976.

Brenner, H., Int. J. Multiphase Flow, 1974, 1, 195-341.

Buxpaum, R.E., "The Separation of Tritjun from the Liquid Lithium BreederBlanket of a Fusion Reactor: The Use of Tritium Metal as a Getter," Ph.o Thesis, Princeton University, Princeton, New Jersey, 1981.

Buxhaum, R.E. and Johnsor, E.F., Nucl. Technol. 1980, 49, 307-314.

Carlson, O.N., Schmidt, F.A. and Peterson, D.T., J. Less Common Metals, 1966, $101-41$.

Cussler, E.L., "Multicomponent Diffusion," Elsever Scientific Fib. Co., New York, 1976, 308-310.

Einstein, A., "Investigations on the Theory of Brownian Movement," translated by A.J. Cowper, Dover Pliblications, Inc., New York, 1955.

Fresius, F., Laharn, H.J., Spalthoff, W., and Wille, P., Berichte der BunsenGesseil, 1972, 76, 1216-1220. 
Fresius, F., Hackbarth, H., and wille, P., Atomkernenergic, 1976, 27, $287-8$.

Jung, J., J. Nucl. Mat. 1973-4, 49, 299-30s.

Langevin, P., C.R. Acad. Sci. 1908, 146, 530-33.

Russel, w.B., Ann. Rev. Fluid Mech. 1981, 13, 425-55.

wilke, Q.R. and Chang, P., AICheE. J. 1955, 1, 264.

Scheibel, E.G., Ind, Engng. Chem. 1954, 46, 2007.

Shpil'rain, E.E., Soldatenko, Y.A., Yakimovich, V.A., Fomin, V.A., Sarchenko, V.A., Belova, A.M., Kagon, D.N. and Dramova, I.F., High Temp. 1965, 3, 870.

Talbot, J.B., and Clinton, S.L., Proceedings of 3th Symposium on Engineering Problems of Fusion Research iInstitute of Electrical and Electronics Engineers, New York, 1979) IEEE Pub. No. 79C41441-5 NPS, Vol. I, pp. $38 \dot{-393 .}$

Talbot, J. AIChr: symp. Ser. 208, 1981, $77,58-63$.

Volkyl, J., and Alefield, G., "Topizs in Applied Physics, Hydrogen in Metals I," Springer Verlag, New York, 1978, pp. 321-348.

Voroboyov, V.A., and Ryalschikov, L., Ukr. Fiz. 2h. 1966, 11, 209-211 (translated by Louis furjes, 1980). 
Zener, C,, "Imperfections in Necrly Perfect Crystals," John Wiley and Sons, Inc., New York, 1950, pp. 289-303. 
FIGURE CAPTIONS

Fig. ? Diffusivity of Hydrogen in Ytrrium. 


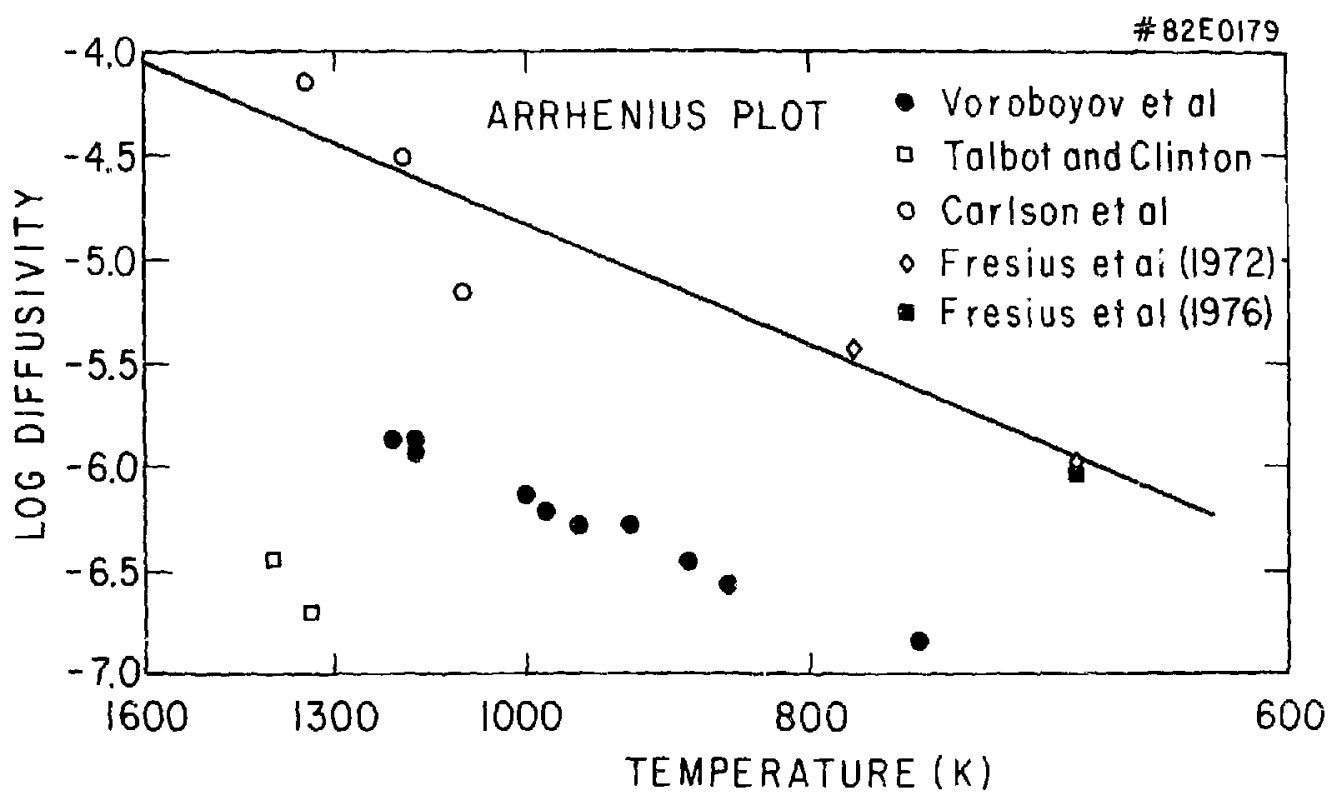

ㄴ. 